\title{
Bacteria Foraging Algorithm in Antenna Design
}

\author{
Biswa Binayak Mangaraj, ${ }^{1}$ Manas Ranjan Jena, ${ }^{2}$ and Saumendra Kumar Mohanty ${ }^{3}$ \\ ${ }^{1}$ Department of Electronics and Telecommunication Engineering, Veer Surendra Sai University of Technology, Sambalpur, \\ Odisha 768018, India \\ ${ }^{2}$ Department of Electronics and Telecommunication Engineering, Synergy Institute of Engineering \& Technology, \\ Dhenkanal, Odisha 759001, India \\ ${ }^{3}$ Department of Electronics and Instrumentation Engineering, Institute of Technical Education \& Research, \\ S 'O’A University, Bhubaneswar, Odisha 751030, India
}

Correspondence should be addressed to Biswa Binayak Mangaraj; bbmangaraj@yahoo.co.in

Received 14 November 2015; Revised 14 January 2016; Accepted 14 January 2016

Academic Editor: Francesco Carlo Morabito

Copyright (C) 2016 Biswa Binayak Mangaraj et al. This is an open access article distributed under the Creative Commons Attribution License, which permits unrestricted use, distribution, and reproduction in any medium, provided the original work is properly cited.

\begin{abstract}
A simple design procedure to realize an optimum antenna using bacteria foraging algorithm (BFA) is proposed in this paper. The first antenna considered is imaginary. This antenna is optimized using the BFA along with a suitable fitness function formulated by considering some performance parameters and their best values. To justify the optimum design approach, one 12-element YagiUda antenna is considered for an experiment. The optimized result of this antenna obtained using the optimization algorithm is compared with nonoptimized (conventional) result of the same antenna to appreciate the importance of optimization.
\end{abstract}

\section{Introduction}

An antenna is an indispensable electromagnetic wave radiating/receiving device in any wireless transreceiving communication system. It can be single structures like monopole, dipole, folded dipole, loop, spiral, helical, and so forth, and complex structures like linear array and planar array of these single structures. These single structures have few and complex structures have many input parameters referred to as design parameters and output parameters referred to as performance parameters. Some of the design parameters of an antenna are the length of the elements $\left(l_{n}, n=1,2, \ldots, N\right)$, radius of the elements $\left(a_{n}, n=1,2, \ldots, N\right)$, specific spacing between neighbor elements $\left(d_{m n}, m=1,2, \ldots,(N-1)\right.$ and $n=2,3, \ldots, N)$, operating frequency $\left(f_{0}\right)$, input supply $\left(V_{i}\right.$ or $\left.I_{i}\right)$, and the orientation of the elements. Similarly, some of the performance parameters of an antenna are input impedance $\left(Z_{\text {IN }}\right)$, directivity $(\mathrm{DR})$, front to back ratio (FTBR), front to maximum side lobe level (FSLL) in E- and H-planes, half-power beamwidth (HPBW) in E- and H-planes, and bandwidth (BW). An antenna designed using theoretical design parameter values, as available in textbooks, do not provide desired performance parameter values. Desired performance parameter values can be achieved, only when the same antenna is optimized, and optimum design parameters are found out. Soft computing tools like Genetic Algorithm (GA), Ant Colony Optimization (ACO), Simulated Annealing Algorithm (SAA), Particle Swarm Optimization (PSO), Differential Evolution (DE), Bacteria Foraging Optimization (BFO), Cuckoo Search (CS), and many others can be used to optimize the design parameters of the antenna [1-13]; only when the proper structure code of the antenna is available. Further, the code of the soft computing tool should be such that it is readily linked to the available structure code. In this regard, the MATLAB application software is very suitable to develop the code of the antenna structure and the soft computing tools.

It is very difficult and also a time-taking procedure to find out the parameters of any antenna using simple intuition, experience, and practical measurements [1]. To get the best design of an antenna within a minimal time, various optimization techniques like Gradient Descent Learning [2], GA $[1,3,4]$, ACO [5], SAA [5, 6], PSO [7, 8], DE [9], BFO [10$13]$, and so forth can be used. So far as optimized results are 
TABLE 1: Design and performance parameters of an imaginary antenna.

\begin{tabular}{lccccc}
\hline Serial number & $\begin{array}{c}\text { Design } \\
\text { parameters }\end{array}$ & $\begin{array}{c}\text { Range of the design } \\
\text { parameters }\end{array}$ & $\begin{array}{c}\text { Performance } \\
\text { parameters }\end{array}$ & $\begin{array}{c}\text { Range of the performance } \\
\text { parameters }\end{array}$ & $\begin{array}{c}\text { Assumed best performance } \\
\text { parameters }\end{array}$ \\
\hline 1 & $D_{A}$ & $0-10$ units & $P_{A}$ & $45-55$ units & 50 units \\
2 & $D_{B}$ & $3-15$ units & $P_{B}$ & $0-45$ units & 0 units \\
3 & $D_{C}$ & $5-10$ units & $P_{C}$ & $30-60$ units & 30 units \\
4 & $D_{D}$ & $1-5$ units & $P_{D}$ & $0-15$ units & 30 units \\
5 & $D_{E}$ & $40-50$ units & $P_{E}$ & $15-30$ units & 5 units \\
6 & $D_{F}$ & $1-2$ units & $P_{F}$ & $0-5$ units & 5 units \\
7 & $D_{G}$ & $0-30$ units & $P_{G}$ & $5-10$ units & 60 units \\
8 & - & - & $P_{H}$ & $60-90$ units & \\
\hline
\end{tabular}

concerned, convergence is better in case of bacteria foraging algorithm (BFA) [12], and the systematic algorithm is strong enough for global optimization [13]. BFA is also suitable to achieve multiparameter, multiobjective, and nonlinear designs through optimization [12]. Though BFA is used in designing the various $\mathrm{V}$ dipoles and Yagi-Udas explained in $[11,12]$, the exact implementation of the BFA in designing the antenna considering the role of biological agents (bacteria) is overlooked. In this paper an appropriate elaboration of BFA is made so far as antenna design is concerned in a step by step manner. The biological agents of the BFA here are responsible for optimum design of the antenna through selection of a suitable fitness function.

As per the requirement of the antenna design through optimization, first of all, we consider some design parameters and some performance parameters recognized by some arbitrary notations for an imaginary antenna which will be modified by the designer while considering a real antenna. A step by step procedure is also described to optimize all the design parameters of the imaginary antenna to get some desired performance parameters. The number of both design and performance parameters can be chosen by the designer. During the optimization process, after each simulation a unique fitness value is obtained for each new design. This fitness value decides the best design and the corresponding best performance parameters. To obtain a fitness value for each new design a suitable fitness function is to be formulated by the designer. Formulation of proper fitness function considering the performance parameters and their range has a major role during the optimization process. In fact, each of the performance parameters of an antenna is a function of all the design parameters of the antenna. The desired result as well as convergence cannot be achieved, only when the selection of fitness function is proper, the ranges of the design along with performance parameters are known, and the best value of the performance parameters are also known. The fitness function has a range of values between $\mathrm{FT}_{\text {MAX }}$ and $\mathrm{FT}_{\mathrm{MIN}}$. If we want to minimize the fitness function for a better design, the best design produces a fitness value close to $\mathrm{FT}_{\mathrm{MIN}}$ and vice versa.

The paper is organized in seven sections. After the introduction in Section 1, Section 2 describes some design and performance parameters and their range in general for any imaginary antenna. Section 3 explains the selection of parameters for the formulation of fitness function and subsequent optimization. Section 4 explains the BFA, its importance, and the exact requirement for the optimization. Antenna design in the light of BFA is explained in Section 5. Section 6 presents the design and performance parameters of a conventional 12-element Yagi-Uda antenna and the same parameters of the same antenna optimized using BFA to show some better performance. Section 7 concludes the paper.

\section{Design and Performance Parameter of an Imaginary Antenna}

In case of any antenna there can be many design parameters and many performance parameters as well. For example, one antenna may have seven design parameters such as $D_{A}, D_{B}, D_{C}, D_{D}, D_{E}, D_{F}$, and $D_{G}$ and eight performance parameters such as $P_{A}, P_{B}, P_{C}, P_{D}, P_{E}, P_{F}, P_{G}$, and $P_{H}$. Furthermore, one of the design parameters, for example, can be length of the nth element $\left(l_{n}\right)$ of the antenna and one of the performance parameters can be directivity (DR) of the same antenna. In Section 1 some of the important design and performance parameters are already mentioned. Each of these design parameters has a range, and within its range at a particular value the antenna performs best. Similarly, each of the performance parameters has also a range, and in general either the minimum or the maximum value decides the best performance. For example, if the DR is maximum for a directive antenna, then it is a better performance parameter and if the beamwidth of the radiation lobe is minimum, then it is also a better performance parameter for the same antenna. In case of very few parameters like input impedance, the midpoint value within the range decides the best performance.

Now, let us select the range and the corresponding best value of the design and performance parameters of the above antenna as shown in Table 1 . The exact units of these design and performance parameters have not been mentioned since the antenna considered here is imaginary. Once a real antenna is considered, subsequently its parameters can be identified whose units can also be mentioned. In Section 6 a real antenna that is a 12-element Yagi-Uda has been considered for optimization. In that section, the units of the design and 
TABLE 2: Range and best value of design and performance parameters considered for Yagi-Uda.

\begin{tabular}{|c|c|c|c|c|c|c|}
\hline \multirow[b]{2}{*}{$\begin{array}{l}\text { Serial } \\
\text { number }\end{array}$} & \multicolumn{3}{|c|}{ Design parameters } & \multicolumn{3}{|c|}{ Performance parameters } \\
\hline & Parameters & $\begin{array}{l}\text { Parameters' range } \\
\text { (actual unit) }\end{array}$ & Best value & Parameters & $\begin{array}{c}\text { Parameters' range } \\
\text { (actual unit) }\end{array}$ & Best value (actual unit) \\
\hline 1 & Length & $0.2-0.6$ units $(\mathrm{m})$ & \multirow{8}{*}{$\begin{array}{l}\text { To be found out } \\
\text { through } \\
\text { optimization }\end{array}$} & Gain & 02-100 units & 100 units \\
\hline 2 & Separation & $0.1-0.5$ units $(\mathrm{m})$ & & Directivity (DR) & 03-20 units $(\mathrm{dB})$ & 20 units $(\mathrm{dB})$ \\
\hline 3 & - & - & & $\operatorname{Real}\left(Z_{\mathrm{IN}}\right)$ & $30-70$ units (Ohms) & 50 units $(\Omega s)$ \\
\hline 4 & - & - & & $\operatorname{Imaginary}\left(Z_{\mathrm{IN}}\right)$ & 00-20 units (Ohms) & 00 units $(\Omega s)$ \\
\hline 5 & - & - & & FTBR & 01-40 units $(\mathrm{dB})$ & 40 units $(\mathrm{dB})$ \\
\hline 6 & - & - & & FSLL & $01-40$ units $(\mathrm{dB})$ & 40 units $(\mathrm{dB})$ \\
\hline 7 & - & - & & EHPBW & 05-70 units (degree) & 05 units $\left(^{\circ}\right)$ \\
\hline 8 & - & - & & HHPBW & 05-70 units (degree) & 05 units $\left(^{\circ}\right)$ \\
\hline
\end{tabular}

the performance parameters are explained and the values are also shown in Table 2.

\section{Formulation of Fitness Function}

Before formulation of the fitness function, first of all one should take a decision: whether it is to be minimized or maximized. If it is decided to minimize the fitness function, subsequently for the best design the fitness value of the fitness function should be as small as possible. When a fitness function is formulated for the optimization process, it is always the performance parameters which are considered. This is because we need to achieve certain desired performance parameters from the antenna under consideration for some specific application. Further, in case of any antenna each of these performance parameters is a function of all the design parameters. Hence, we can write

$$
\begin{aligned}
& P_{A}=f_{1}\left\{D_{A}, D_{B}, D_{C}, D_{D}, D_{E}, D_{F}, D_{G}\right\}, \\
& P_{B}=f_{2}\left\{D_{A}, D_{B}, D_{C}, D_{D}, D_{E}, D_{F}, D_{G}\right\}, \\
& P_{C}=f_{3}\left\{D_{A}, D_{B}, D_{C}, D_{D}, D_{E}, D_{F}, D_{G}\right\}, \\
& P_{D}=f_{4}\left\{D_{A}, D_{B}, D_{C}, D_{D}, D_{E}, D_{F}, D_{G}\right\}, \\
& P_{E}=f_{5}\left\{D_{A}, D_{B}, D_{C}, D_{D}, D_{E}, D_{F}, D_{G}\right\}, \\
& P_{F}=f_{6}\left\{D_{A}, D_{B}, D_{C}, D_{D}, D_{E}, D_{F}, D_{G}\right\}, \\
& P_{G}=f_{7}\left\{D_{A}, D_{B}, D_{C}, D_{D}, D_{E}, D_{F}, D_{G}\right\}, \\
& P_{H}=f_{8}\left\{D_{A}, D_{B}, D_{C}, D_{D}, D_{E}, D_{F}, D_{G}\right\} .
\end{aligned}
$$

Now, we see from Table 1 that the best performance parameters are $P_{\text {Abest }}=50, P_{B \text { best }}=0, P_{\text {Cbest }}=30, P_{\text {Dbest }}=$ $15, P_{\text {Ebest }}=30, P_{\text {Fbest }}=5, P_{\text {Gbest }}=5$, and $P_{\text {Hbest }}=$ 60. Considering all the performance parameters and their corresponding best values (desired performance parameters) the fitness function can be formulated as follows:

$$
\begin{aligned}
\mathrm{FT}(P)= & a\left|P_{A}-P_{\text {Abest }}\right|+b\left|P_{B}-P_{B \text { best }}\right| \\
& +c\left|P_{C}-P_{\text {Cbest }}\right|+d\left|P_{D}-P_{\text {Dbest }}\right|
\end{aligned}
$$

$$
\begin{aligned}
& +e\left|P_{E}-P_{\text {Ebest }}\right|+f\left|P_{F}-P_{\text {Fbest }}\right| \\
& +g\left|P_{G}-P_{\text {Gbest }}\right|+h\left|P_{H}-P_{\text {Hbest }}\right|
\end{aligned}
$$

where $a, b, c, d, e, f, g$, and $h$ are scalar constants whose values are $0.025,0.00277,0.00416,0.00833,0.00883,0.025$, 0.025 , and 0.00416 , respectively. These values are found out by considering equal weightage to all the eight objective functions and the worst case values of the eight performance parameters. In such a case variation of fitness value is in the range of 0 and 1 . If we decide in particular that the performance parameter " $P_{\mathrm{C}}$ " is of greater importance, where we are interested in giving 50 percentage weightage to this parameter and equal weightage to the rest of objective functions, then all the above scalar constants are changed. The new values of the scalar constants, respectively, are 0.01428 , $0.001587,0.01666,0.00476,0.00476,0.01428,0.01428$, and 0.00238. The fitness function considered here as in (2) will provide the best design or the worst design, when the fitness value of the fitness function is close to 0 (zero) or 1 (one), respectively, as per the algorithm of BFA.

\section{Bacteria Foraging Algorithm and Optimization}

Bacteria foraging algorithm (BFA) is a powerful optimization method that is formulated by considering the elimination of creatures with poor food searching tactics. The poor searching strategy can be reshaped into better strategy. Even though there are several optimization methods, BFA is regarded as a powerful algorithm to deal with global optimization problems [13], due to its inbuilt superiority so far as convergence time [12], strength, and precision are concerned. The cause of lesser consumption time is owing to the cognitive and sensing capability of the bacterium and the duration is hardly changed even though many parameters are optimized. The BFA as a biomimicry of the foraging strategy is established by Passino [14] to optimize several problems in control system [15]. Because of the benefits as indicated, another electrical engineering problem has also been optimized using BFA [13]. In dealing with antenna optimization problems with the BFA, it is of utmost importance to formulate a fitness 
function which is basically multiparameter, multiobjective, and nonlinear in nature. From a biological point of view, the term foraging is considered as an optimization technique with a thought that the work done by the bacterium in searching the amount of food per unit time should be as small as feasible. Thus, a fitness function would be there with some objectives as a measure of the work done by the bacterium in seeking the nutrient. The work done can also be interpreted as the cost observed by the bacterium during the process of searching nutrient. Therefore, the primary objective of the $\mathrm{BFA}$ is to make the cost incurred to be as small as possible. The whole procedure in attaining the small cost considers four unique biological steps, that is, chemotaxis, swarming, reproduction, and elimination-dispersal [13, 16].

In the chemotaxis stage, two types of motions of the bacteria are involved, that is, swim and tumble. These movements of bacteria are due to the rotary motion of the flagellum available with each bacterium. During a searching practice, if the bacterium gets sufficient food, the rotary motions of all flagella occur in an anticlockwise direction and consequently it swims to another location in order to find still more food. On the contrary, if the amount of food is less, the bacterium tumbles due to clockwise rotary motion of all flagella. The following step is swarming which is distinguished by the collective action of all the bacteria. Bacteria with ample food discharge some chemicals named as an attractant to communicate the distant week bacteria regarding the availability of abundant nutrient at their place. The swarming activity depends on two parameters such as width of attractant and depth of attractant. The width of attractant is associated with a diffusion rate of the attractant. The depth of attractant decides the influence range up to which attractant continues so that information regarding the place of nutrients is accurately intimated. The original bacteria, who have invited the weak bacteria, set a height of repellent and hence never allow them to eat their food. The width of the repellent is generally related to common activities of the bacterium. Respectively, the defined values of the width and depth of attractant, width, and height of repellent are $0.2,0.1,10$, and 0.1 as in [15]. The next step is the reproduction one which is performed with the splitting of bacterium into two, maintaining the bacteria count to be fixed. In this step discrimination is made between healthy bacterium and weak bacterium so that healthy bacterium is split into two bacteria in the same position while weak one dies. The final step, that is, elimination-dispersal, is influenced by other conditions like acidity, alkalinity, temperature, absence or presence of water, and so forth. The bacteria may be eliminated in an inappropriate condition or dispersed to new locations owing to water push. The last event avoids the stagnant condition.

When an antenna is to be optimized to achieve some desired performance parameters, using a soft computing tool like BFA, then the codes of the antenna structure and the soft computing tool are required. It is always better if both of the codes are written using the same computer language. MATLAB application software is well known and easy to implement in this regard. Once both codes are available, the optimization process can be initiated. Before optimization, both the codes are to be properly linked, where optimization code performs as the main program and the structure code performs as subroutine program. During the optimization process when the structure code is run, a fitness value is obtained. A new fitness value is obtained for each new design (during the subsequent run), which is supplied to the optimization code through function call. The fitness value is evaluated by fitness function available at the end of the structure code, after execution of the structure code program and subsequent evaluation of all the performance parameters. At the end of the optimization process, the soft computing tool provides the desired performance parameters and the corresponding design parameters basing on the algorithm of the soft computing tool.

\section{Antenna Design in the Light of BFA}

The range of design parameters within which antenna performs must be known to the designer. This knowledge of range is essential for the optimization point of view so that optimization algorithm can be remodeled for effective use. Basically the bacteria are greedy, as they always search and consume food till their last breath. So this searching activity can be modeled to search better parameter. Thus, swim, tumble, swarm, and dispersal and elimination activity of bacteria can be modeled to achieve best parameters of the antenna design.

The biological activities of bacteria can be understood by the behavior of $E$. coli bacterium [14, 15]. This bacterium principally undertakes two kinds of motions, that is, swimming and tumbling. A typical chemotactic behavior of E. coli bacterium is shown in Figure 1.

In this example a single $E$. coli bacterium initiates its movement from starting position. The symbols for flagella rotation have been identified in the inset. At the starting position the symbol is for counterclockwise, and hence the bacterium moves to the second position by swimming. The same argument is applicable for the movement from second to third position. In the third position, the flagella exhibit the symbol for clockwise rotation. Hence the movement from third to fourth position is through the process of tumble. Similar explanation is applicable for the other positions.

The movement features of bacterium in BFA, such as swimming, tumbling, and swarming are accountable for the change in design parameters (i.e., $D_{A}, D_{B}, D_{C}, \ldots$ ) of any antenna structure in order to make the fitness values of the fitness functions $\mathrm{FT}(P)$ as small as possible and thus producing BFA optimized antenna to attain all the performance parameters of course within reasonable computational time.

Figure 2 explains the chemotaxis of bacteria in association with the optimization of the above antenna. In this optimization problem, four bacteria are considered for simplicity. These bacteria are placed at four random positions and normally take almost equal numbers of chemotactic steps during their life span. Every location in the problem space has a unique value of fitness, as each case intimates the physical condition of the bacterium; the lesser is the value due to the performance parameters, the healthier is the bacterium. 


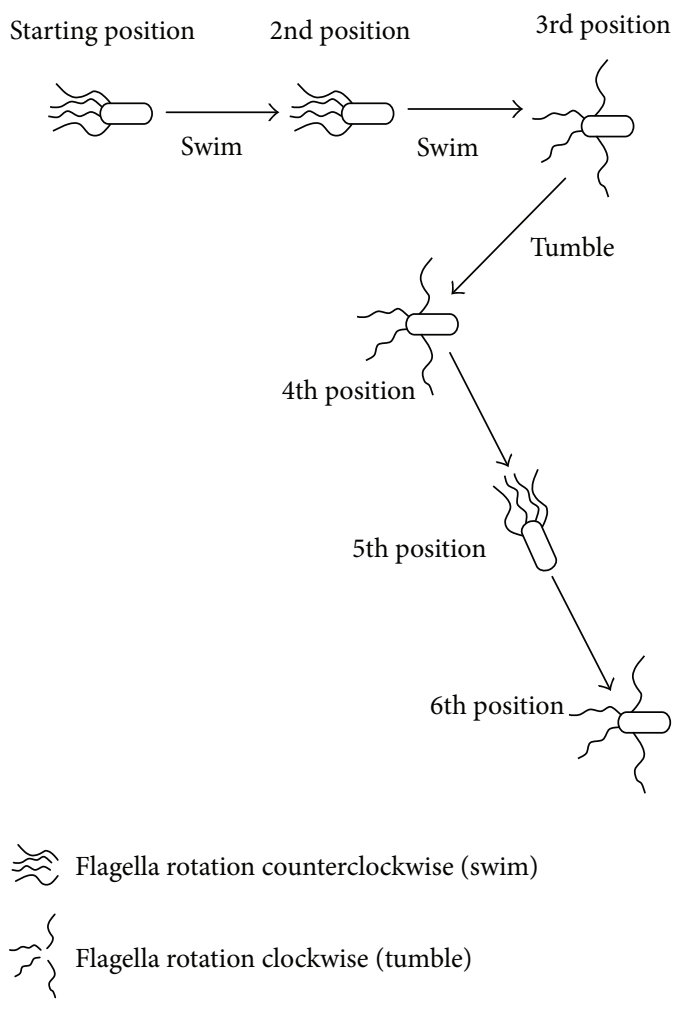

Figure 1: Chemotactic behavior of E. coli bacterium.

The fitness of the 1st bacterium at the initial location is designated as $\mathrm{FT}_{11}^{\mathrm{OO}}$. The representations considered here have some distinctive sense.

The preferred symbol of the fitness function in terms of antenna performance parameters is as shown in Figure 2 and is represented by

$$
\operatorname{FT}_{j i}^{p q}\left(P_{j i}^{p q}\right)
$$

where $j$ is the number of the bacteria, 1, 2, 3, and 4, $i$ is the position of the bacteria, $1,2, \ldots, 10$, and $p$ is the type of motion by bacterium to arrive at $(i-1)$ th position. $q$ is the type of motion by bacterium to arrive at $i$ th position:

$$
p, q=\left\{\begin{array}{l}
S \text { for swim, } \\
T \text { for tumble, } \\
O \text { for initial condition. }
\end{array}\right.
$$

As per biology, the bacteria attain their fitness on the basis of the amount of nutrients they consume. However, in the antenna domain the fitness is found using (2) with the specific values of performance parameters (i.e., $P_{A}, P_{B}, P_{C}, \ldots$ ) which are found by taking the suitable values of the antenna design parameters (i.e., $D_{A}, D_{B}, D_{C}, \ldots$ ) of any antenna. As the bacterium changes its position, the value of fitness is also altered. At each $i$ th new position, the value of fitness is compared with that of $(i-1)$ th position. Basing on the value, either swimming or tumbling motion is initiated at the present position. Accordingly, if $\mathrm{FT}_{j i}^{p q} \leq \mathrm{FT}_{j(i-1)}^{p q}$, the design parameters get modified as given by (5), and thereby all performance parameters of the antenna get modified as given by (6) following the swim movement:

$$
\begin{aligned}
& D_{j i}^{p q}=D_{j(i-1)}^{p q}+\Delta_{S} \times D_{j(i-1)}^{p q}, \\
& P_{j i}^{p q}=P_{j(i-1)}^{p q}+\Delta_{S} \times k\left(f_{n}\right) \times P_{j(i-1)}^{p q},
\end{aligned}
$$

where $\Delta_{S}$ is taken as 0.03 to perform the computation within a small time. However, if $\mathrm{FT}_{j i}^{p q}>\mathrm{FT}_{j(i-1)}^{p q}$, a tumble movement makes all the design parameters $D_{j i}^{p q}$ of the antenna to take any random value, from the predefined range of the design parameters of the antenna [17].

The 1st movements of bacteria are always the swim, as there is no value of the fitness function at the starting position. Therefore, the fitness value at the 2 nd position of the 1st bacterium is symbolized by $\mathrm{FT}_{12}^{\mathrm{OS}}$ or $\mathrm{FT}_{12}^{S S}$. With the change of position from 2 nd to 3 rd one, the fitness value is represented as either $\mathrm{FT}_{13}^{S S}$ or $\mathrm{FT}_{13}^{S T}$ basing upon the type of movement. The other bacterium also observes the same taxonomy. At the initial place, the fitness is symbolized as $\mathrm{FT}_{11}^{\mathrm{OO}}$ and the relevant value of the design parameter is $D_{11}^{O O}$. The first antenna design is accomplished with this first group of design parameters and thereby generating the first fitness value $\mathrm{FT}_{11}^{\mathrm{OO}}$. As the bacteria change their position, a number of design parameters of the antenna also get changed and consequently a new antenna is obtained with a fresh fitness value for each. In the proposed BFA optimized antenna design process, one swarming process is taken up after every four numbers of chemotaxis to minimize the computational time. From the biological perspective, the decline in antenna design time is revealed by the method of information communication to signal the hungry bacteria about the place of rich nutrient. After every swarming process the chemotaxis continue. Total number of chemotactic steps decides the number of swarming processes. In the BFA code implemented for the antenna designs, the swarming activity starts after completion of required chemotactic steps and with a middle value, that is, 0.5 for the fitness function. The fitness functions with values less than 0.5 are unchanged while those more than or equal to 0.5 are replaced with a lowest fitness value. At the end the best design was provided by second bacterium at fourth place with a lowest fitness value of $\mathrm{FT}_{24}^{\mathrm{SS}}$ as shown in Figure 3.

The reproduction or generation step begins after the chemotaxis and swarming step. In this cycle, 50\% of the total antennas designed are rejected basing on poorer fitness values, whereas the rest $50 \%$ go through a reproductive phase with replication of fitness value in order to keep the bacteria count (antenna designs) unchanged. After completion of each reproduction step the chemotaxis and swarming are initiated again. As the generation or reproduction stage is finished, the antenna design process enters the final stage, that is, elimination or dispersal. During this stage, $25 \%$ of the total antennae designed are either rejected or redesigned. The rejection is based on very poor values of the fitness while the redesigned is carried out with random values of all the design parameters of the antenna within the desired range 


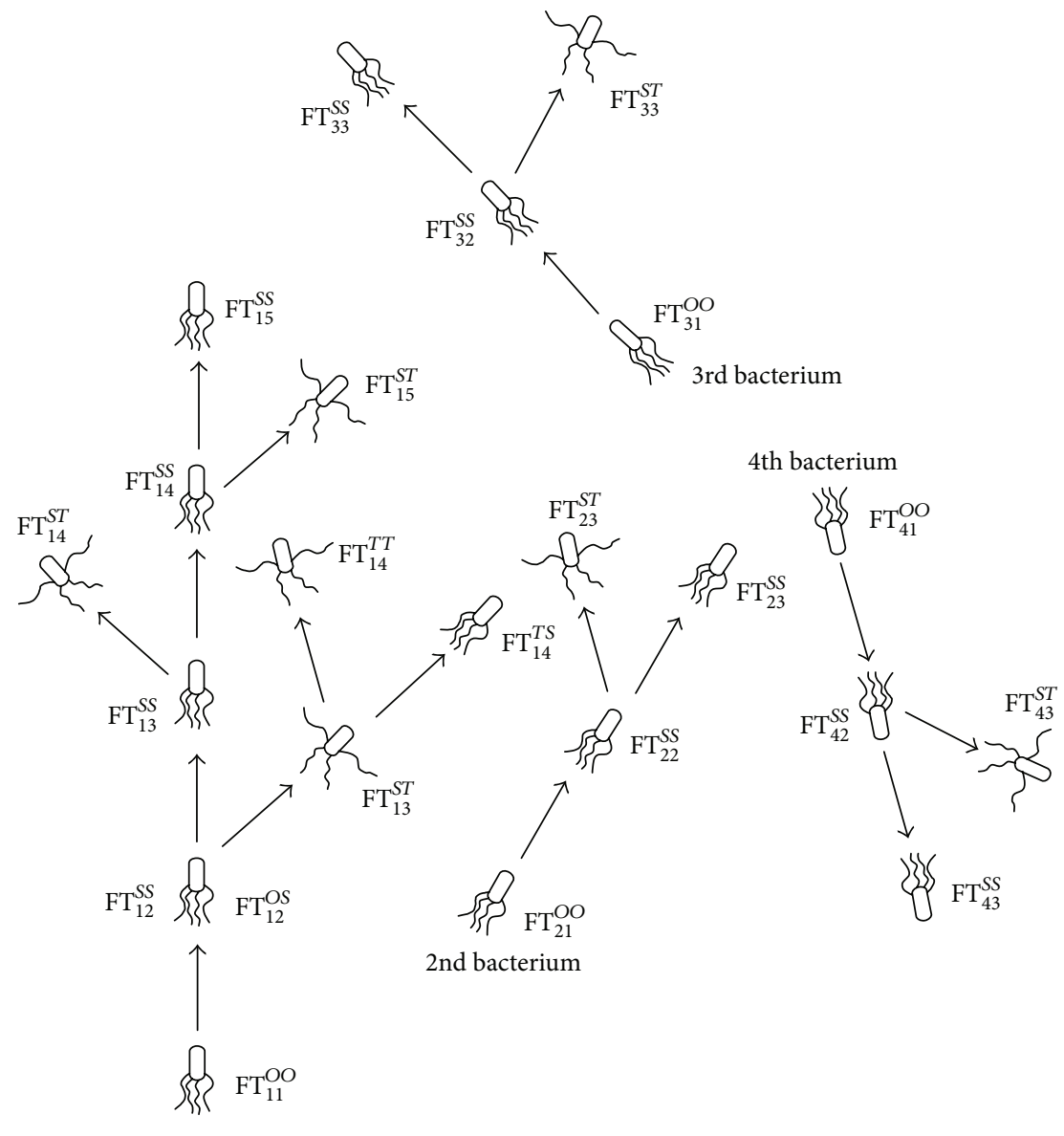

1st bacterium

FIgURE 2: Chemotaxis of bacteria in the light of antenna optimization using BFA.

of respective parameters. The remaining $75 \%$ designs remain the same. The whole process is continued again. The above biological stages associated with BFA have been referred to in [12].

\section{A Typical Design with and without Optimization}

Figure 4 represents the generalized structure of $N$ element Yagi-Uda structure. The physical dimensions of this antenna are systematic in nature as explained in [17]. The arrangement of the dipoles, the feeding to only one active element (driver, second dipole from left) is as shown in Figure 4 and has a major role in radiation. Out of all the dipoles of this antenna the largest passive dipole at its left end acts as an inductive element and so as a reflector [17]. All the rest dipoles which are passive in nature act as capacitive elements and so as directors [17]. This kind of arrangement is the major reason of directive nature of Yagi-Uda.

The design parameters of this antenna which are to be optimized are as follows:

$$
\begin{aligned}
& N=12, \\
& l_{1}=\text { length of the reflector, }
\end{aligned}
$$

$l_{2}=$ length of the active element,

$l_{3}=$ length of the 1st director,

$l_{n}=$ length of $(n-2)$ th director,

$l_{12}=$ length of the 10th director,

$d_{12}=$ distance between the reflector and the active element,

$d_{23}=$ distance between the active element and the 1st director,

$d_{(n-1) n}=$ distance between the $(n-3)$ th and the $(n-$ 2) th directors,

$d_{1112}=$ distance between 9 th and 10th directors,

$a_{0}=$ radius of the wire.

A conventional 12-element Yagi-Uda antenna design requires a driven element of total length $\left(l_{2}\right)$ little less than $0.5 \lambda$ [17], say $10 \%$ less, so close to $0.49 \lambda$. The reflector's length $\left(l_{1}\right)$ of the same antenna needs to be more than $0.5 \lambda$ [17], say $10 \%$ more, so close to $0.51 \lambda$. The length of the directors $\left(l_{3}, l_{4}, l_{5}, \ldots, l_{12}\right)$ of the same antenna needs to be less than the driven element and should be in the range of $0.45 \lambda$ to $0.40 \lambda$ [17], so approximately $0.450 \lambda, 0.445 \lambda, 0.440 \lambda, 0.435 \lambda, 0.430 \lambda$, $0.425 \lambda, 0.420 \lambda, 0.415 \lambda, 0.410 \lambda$, and $0.400 \lambda$, respectively. 


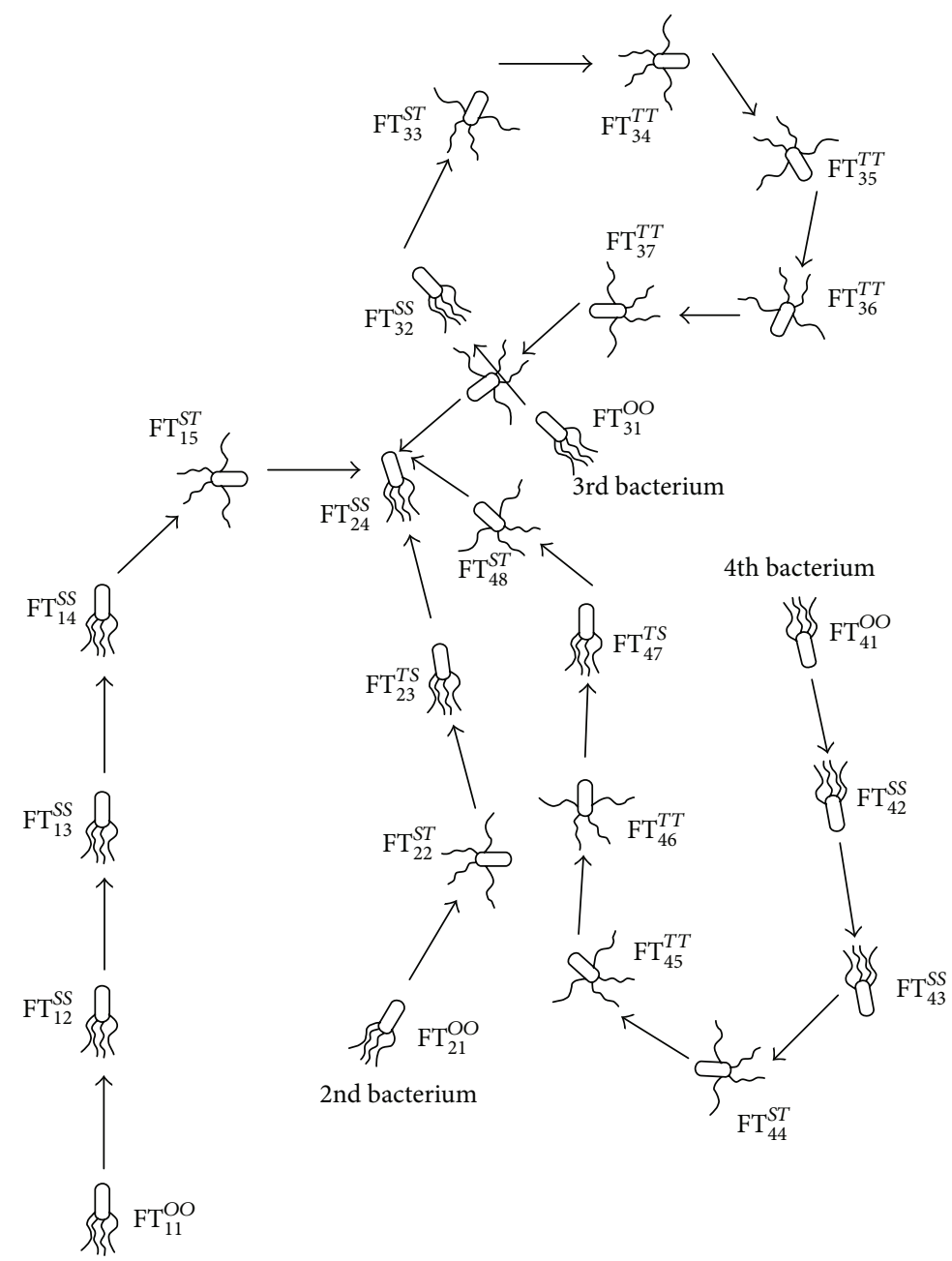

1st bacterium

FIGURE 3: Swarming activity of bacteria in the light of antenna optimization using BFA.

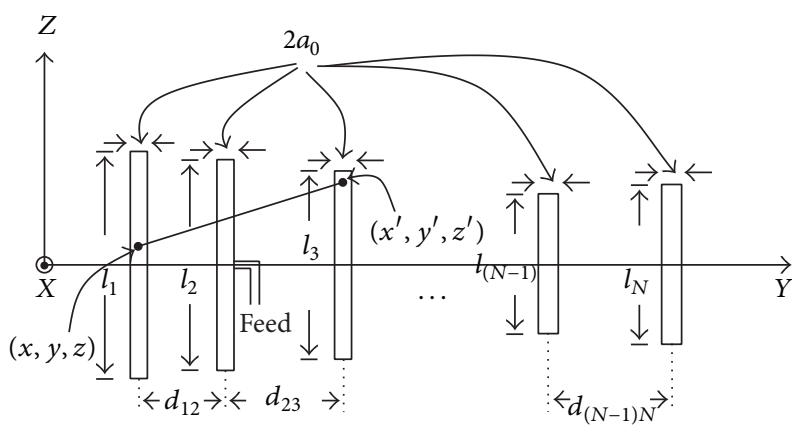

Figure 4: Typical $N$ element Yagi-Uda Array.

The separations between two dipoles need to be kept within the range of $0.3 \lambda$ to $0.4 \lambda$ [17]. Considering the same separation distances for the group of two nearby elements as $0.30 \lambda$, except for the separation between the reflector and the driven element which is kept at $0.25 \lambda$ for optimum design as per the suggestion in [17] and other design parameter as indicated, the Yagi-Uda is designed through simulation and the performance parameters are as shown in Table 3. This is considered as conventional Yagi-Uda. In the same table a 12-element Yagi-Uda optimized using BFA is also shown. The performance parameters provided by this optimized antenna are much better than the conventional design. This indicates that to achieve best performance parameters the same antenna must be optimized using suitable optimization technique.

Before the Yagi-Uda as cited above being optimized, first of all, the range of all its design and performance parameters must be specified. At the same time the best performance parameters have also to be mentioned. Table 2 indicates all the parameters, their range, and the best values.

In this design the unit of the length and the spacing parameters are in meters. This design will operate at an operating frequency of $300 \mathrm{MHz}$. The gain is unitless, whereas the units of DR, $Z_{\mathrm{IN}}$, FTBR, FSLL, EHPBW (HPBW in E-plane), and HHPBW (HPBW in H-plane) are $\mathrm{dB}, \mathrm{Ohms}, \mathrm{dB}, \mathrm{dB},{ }^{\circ}$, and ${ }^{\circ}$, respectively. 
TABLE 3: Design and performance parameters for the Yagi-Uda at $300 \mathrm{MHz}$ with and without BFA optimization.

\begin{tabular}{|c|c|c|c|c|c|c|}
\hline \multirow{3}{*}{$\begin{array}{l}\text { Element } \\
\text { number }(n)\end{array}$} & \multirow{2}{*}{\multicolumn{3}{|c|}{$\begin{array}{c}\text { 12-element Yagi-Uda (conventional) } \\
\text { Fixed design parameters: } a_{0}=0.0032512 \lambda, l / D=100 \\
f_{0}=300 \mathrm{MHz} \\
\text { Optimized design parameters }\end{array}$}} & \multirow{2}{*}{\multicolumn{3}{|c|}{$\begin{array}{c}\text { 12-element Yagi-Uda (optimized) } \\
\text { Fixed design parameters: } a_{0}=0.0032512 \lambda, l / D=100 \text {, } \\
f_{0}=300 \mathrm{MHz} \\
\text { Optimized design parameters }\end{array}$}} \\
\hline & & & & & & \\
\hline & Length $l_{n}$ in $\mathrm{m}$ & Spacing $d_{m n}$ in $\mathrm{m}$ & SWG & Length $l_{n}$ in $\mathrm{m}$ & Spacing $d_{m n}$ in $\mathrm{m}$ & SWG \\
\hline 1 & 0.510 & & 10 & 0.492 & - & 10 \\
\hline 2 & 0.490 & 0.250 & 10 & 0.487 & 0.226 & 10 \\
\hline 3 & 0.450 & 0.300 & 10 & 0.441 & 0.228 & 10 \\
\hline 4 & 0.445 & 0.300 & 10 & 0.422 & 0.206 & 10 \\
\hline 5 & 0.440 & 0.300 & 10 & 0.428 & 0.226 & 10 \\
\hline 6 & 0.435 & 0.300 & 10 & 0.426 & 0.448 & 10 \\
\hline 7 & 0.430 & 0.300 & 10 & 0.424 & 0.396 & 10 \\
\hline 8 & 0.425 & 0.300 & 10 & 0.422 & 0.372 & 10 \\
\hline 9 & 0.420 & 0.300 & 10 & 0.424 & 0.452 & 10 \\
\hline 10 & 0.415 & 0.300 & 10 & 0.416 & 0.434 & 10 \\
\hline 11 & 0.410 & 0.300 & 10 & 0.420 & 0.446 & 10 \\
\hline \multirow[t]{2}{*}{12} & 0.400 & 0.300 & 10 & 0.428 & 0.326 & 10 \\
\hline & \multicolumn{3}{|c|}{ Performance parameters } & \multicolumn{3}{|c|}{ Performance parameters } \\
\hline 1 & \multicolumn{3}{|c|}{$Z_{\mathrm{IN}}(\Omega \mathrm{s})=46.0734+j 3.7654$} & \multicolumn{3}{|c|}{$Z_{\mathrm{IN}}(\Omega s)=50.0075+j 1.0506$} \\
\hline 2 & \multicolumn{3}{|c|}{$\mathrm{DR}(\mathrm{dB})=12.670(+y$-axis $)$} & \multicolumn{3}{|c|}{$\mathrm{DR}(\mathrm{dB})=16.3391(+y$-axis $)$} \\
\hline 3 & \multicolumn{3}{|c|}{$\operatorname{FTBR}(\mathrm{dB})=10.3248$} & \multicolumn{3}{|c|}{$\operatorname{FTBR}(\mathrm{dB})=18.2209$} \\
\hline 4 & \multicolumn{3}{|c|}{ FSLL $(\mathrm{dB})=8.9446$} & \multicolumn{3}{|c|}{ FSLL $(\mathrm{dB})=12.3298$} \\
\hline 5 & \multicolumn{3}{|c|}{ EHPBW $\left({ }^{\circ}\right)=29.1124$} & \multicolumn{3}{|c|}{ EHPBW $\left(^{\circ}\right)=24.6027$} \\
\hline \multirow[t]{2}{*}{6} & \multicolumn{3}{|c|}{ HHPBW $\left(^{\circ}\right)=30.5694$} & \multicolumn{3}{|c|}{ HHPBW $\left({ }^{\circ}\right)=25.3909$} \\
\hline & \multicolumn{3}{|c|}{ Fitness value } & \multicolumn{3}{|c|}{ Fitness value } \\
\hline FT & \multicolumn{3}{|c|}{0.4008} & \multicolumn{3}{|c|}{0.2361} \\
\hline
\end{tabular}

Considering performance parameters and their best values a suitable fitness function is formulated to achieve the best possible design and performance parameters through simulation. The fitness function is as indicated in the following:

$$
\begin{aligned}
\mathrm{FT}(x)= & a|\mathrm{DR}-20|+b\left|\operatorname{Re}\left(Z_{\mathrm{IN}}\right)-50\right| \\
& +c\left|\operatorname{Im}\left(Z_{\mathrm{IN}}\right)\right|+d|\mathrm{EHPBW}-5| \\
& +e|\mathrm{HHPBW}-5|+f|\mathrm{FTBR}-40| \\
& +g|\mathrm{FSLL}-40|
\end{aligned}
$$

where $a, b, c, d, e, f$, and $g$ are $0.0294,0.004167,0.004167$, $0.00152,0.00152,0.00128$, and 0.00128 . The scalar constant values are considered by taking into account highest weightage to DR and other parameters are of equal importance as explained earlier in Section 2. Each of the performance parameters such as DR, $Z_{\mathrm{IN}}$, EHPBW, HHPBW, FTBR, and FSLL are actually functions of all the design parameters, that is, $\left\{l_{1}, l_{2}, l_{3}, \ldots, l_{N}, d_{12}, d_{23}, \ldots, d_{(N-1) N}, a_{0}, N\right\}$. In this expression $l_{n}$ is the length of the $n$th element, $d_{m n}$ is the spacing between two nearby elements ( $m$ th and $n$th element), $a_{0}$ is the radius of elements (fixed), and $N$ is the numbers of dipole elements. The worst value of fitness function is 1 (one) and best value of fitness function is 0 (zero).

The lengths and spacings of various dipoles of a 12element Yagi-Uda array for higher DR are close to $20 \mathrm{~dB}, Z_{\mathrm{IN}}$ close to $50 \Omega$ s, FTBR and FSLL close to $40 \mathrm{~dB}$, and EHPBW and HHPBW close to $5^{\circ}$, simulated using BFA code as the main program and structure code as the function program and the corresponding $Z_{\mathrm{IN}}$, DR, EHPBW, HHPBW, FTBR, and FSLL in E-plane are obtained as shown in Table 3. The E-plane and the H-plane radiation patterns of conventional Yagi-Uda are shown in Figures 5(a) and 6(a) and the E-plane and the $\mathrm{H}$-plane radiation patterns of optimized Yagi-Uda are shown in Figures 5(b) and 6(b). The E-plane and the $\mathrm{H}$ plane radiation patterns of Yagi-Uda are highly directive as expected and maximum field intensity is in the direction of $y$-axis as shown in Figures 5(b) and 6(b) for optimized Yagi$\mathrm{Uda}$, whereas the E-plane and $\mathrm{H}$-plane radiation patterns of conventional Yagi-Uda are with inferior DR as shown in Figures 5(a) and 6(a).

The DR of optimized Yagi-Uda is found to be $16.3391 \mathrm{~dB}$ along the $+y$-axis direction, whereas that for conventional Yagi-Uda is found to be $12.670 \mathrm{~dB}$ along the $+y$-axis direction at $300 \mathrm{MHz}$, that is, the operating frequency at which the 


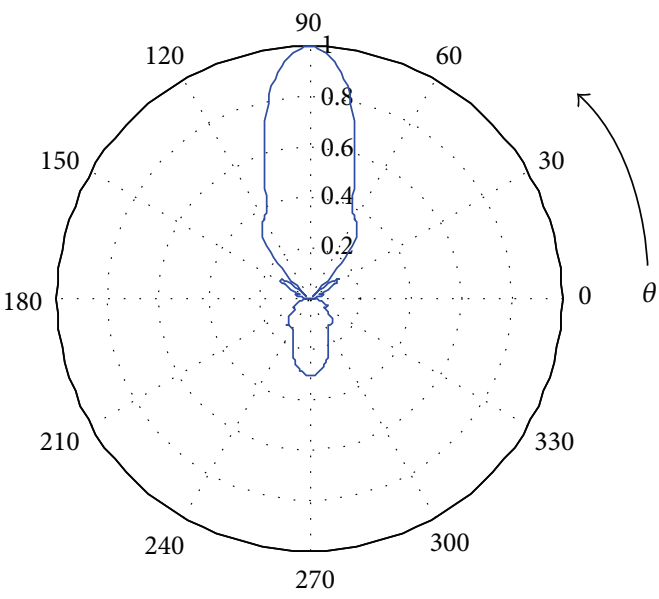

(a) For $\phi=90^{\circ}$

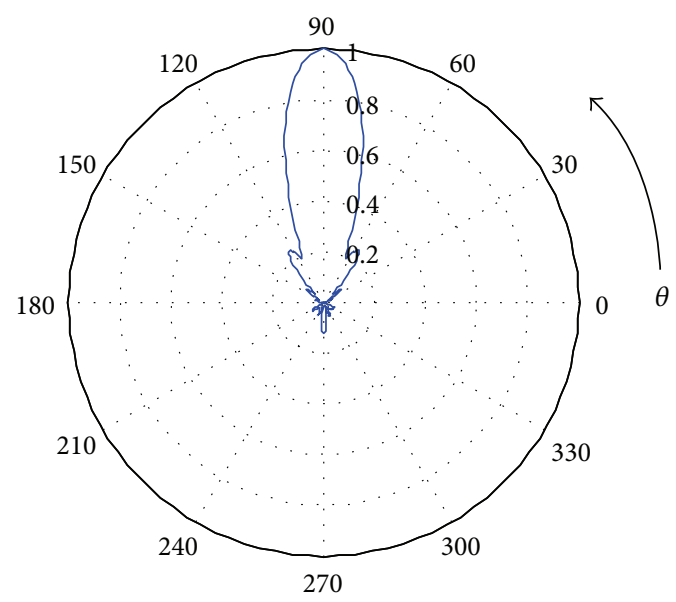

(b) For $\phi=90^{\circ}$

FIGURE 5: Normalized E-plane pattern of 12-element antenna (a) conventional Yagi-Uda and (b) BFA optimized Yagi-Uda.

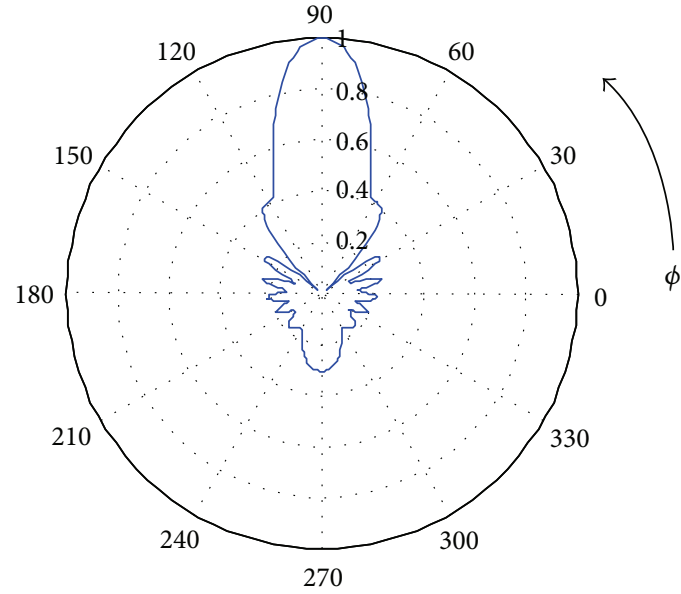

(a) For $\theta=90^{\circ}$

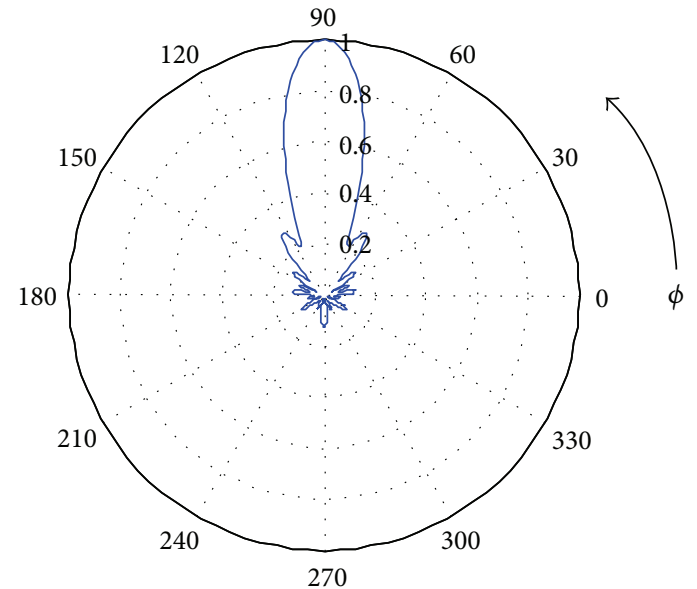

(b) For $\theta=90^{\circ}$

FIGURE 6: Normalized H-plane pattern of 12-element antenna (a) conventional Yagi-Uda and (b) BFA optimized Yagi-Uda.

design is carried out. Hence, there is an increment of $3.669 \mathrm{~dB}$ in the DR which is good enough for a directive antenna and the optimum design suggested in [17] is inferior to this.

The $Z_{\text {IN }}$ of optimized Yagi-Uda is found to be $50.0075+$ $j 1.0506 \mathrm{ohms}$, whereas that for conventional Yagi-Uda is found to be $46.0734+j 3.7654$ at $300 \mathrm{MHz}$. Here also, as per our requirement, the $Z_{\mathrm{IN}}$ is close to $50 \Omega$ s for our optimized case.

The FTBR of optimized Yagi-Uda is found to be $18.2209 \mathrm{~dB}$, whereas that for conventional Yagi-Uda is found to be $10.3248 \mathrm{~dB}$ at $300 \mathrm{MHz}$. The FTBR of conventional YagiUda seems to be poor. The main reason behind this is that the power is not exactly radiated in one direction which is observed from Figures 5 and 6.

The FSLL of optimized Yagi-Uda is found to be $12.3298 \mathrm{~dB}$, whereas that for conventional Yagi-Uda is found to be 8.9446 at $300 \mathrm{MHz}$.
The EHPBW and HHPBW of optimized Yagi-Uda are found to be $24.6027^{\circ}$ and $25.3909^{\circ}$, respectively, whereas those for conventional Yagi-Uda are found to be $29.1124^{\circ}$ and $30.5694^{\circ}$, respectively, at $300 \mathrm{MHz}$. This is observed from Figures 5 and 6.

The design of Yagi-uda using BFA is obtained in a computational environment using Intel $^{\circ} \mathrm{Core}^{\mathrm{ru}} 2$ duo processor T7500 (2.2 GHz, $800 \mathrm{MHz}$ FSB, 4 MB L2 cache) with $2 \mathrm{~GB}$ RAM. The structure codes together with optimization codes are developed in MATLAB 7.2 and linked to each other for the optimization process. The time consumed by the Antenna code for a single run is 4.344 seconds. But the optimization code (with 1 bacterium, 1 chemotactic step, 1 swim step, and 1 reproduction step) and Antenna code for single run consumes a time of 16.610 seconds. Further, the time consumed by the optimization code (with 4 bacteria, 4 chemotactic steps, 4 swim steps, and 1 reproduction step) 


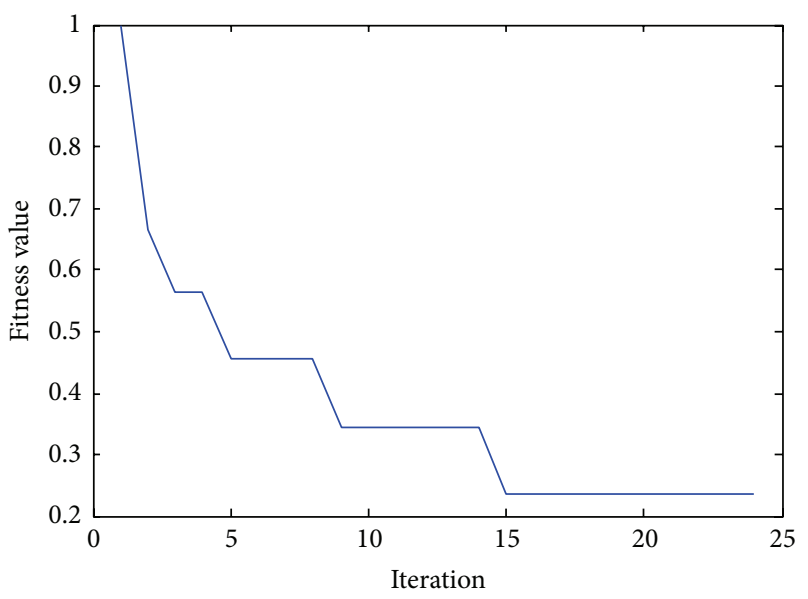

FIgURE 7: Convergence graph of BFA.

and Antenna code is 63.672 seconds. After a number of runs, It is observed that the number of chemotactic steps is in favor of the optimization. Hence, the number of chemotactic steps is taken as 6 and for the design of optimum 12element Yagi-Uda the time consumed by the optimization code (with 4 bacteria, 6 chemotactic steps, 4 swim steps, and 1 reproduction step) and the Antenna code is 98.015 seconds.

After some 15 numbers of iterations out of a total of 24 iterations (Figure 7), the best performance parameters of the optimized Yagi-Uda are obtained as shown in Table 3. The performance parameters as shown in Table 3 indicate optimized Yagi-Uda is far better than the conventional YagiUda. The optimized result obtained in the present case is better than the result provided in $[12,18]$.

\section{Conclusion}

In this paper a simple and effective procedure of antenna design using BFA is explained in a step by step manner. The optimum design of the 12-element wire Yagi-Uda is explained by following the same design procedure. It is observed from Table 3 that the optimization process is highly essential to achieve a better design with better performance parameters. Using the same procedure other wire or microstrip antennas can be optimized and subsequently can be designed. The success in optimum antenna design entirely depends on the knowledge of a suitable fitness function, design and performance parameters of the antenna. The ranges of these design and performance parameters are also equally important which one designer should know.

\section{Conflict of Interests}

The authors declare that there is no conflict of interests regarding the publication of this paper.

\section{References}

[1] E. E. Altshuler and D. S. Linden, "Wire-antenna designs using genetic algorithms," IEEE Antennas and Propagation Magazine, vol. 39, no. 2, pp. 33-43, 1997.

[2] P. Baldi, "Gradient descent learning algorithm overview: a general dynamical systems perspective," IEEE Transactions on Neural Networks, vol. 6, no. 1, pp. 182-195, 1995.

[3] B. B. Mangaraj, S. Hota, and S. K. Mohanty, "Comparative study of two optimizing techniques: bacteria forging algorithm and genetic algorithm in solving V-dipole antenna problem," International Journal of Research in Advance Technology in Engineering, vol. 1, no. 2, pp. 6-15, 2013.

[4] Y. C. Chung and R. L. Haupt, "Amplitude and phase adaptive nulling with a genetic algorithm," Journal of Electromagnetic Waves and Applications, vol. 14, no. 5, pp. 631-649, 2000.

[5] C. M. Coleman, E. J. Rothwell, and J. E. Ross, "Investigation of simulated annealing, ant-colony optimization, and genetic algorithms for self-structuring antennas," IEEE Transactions on Antennas and Propagation, vol. 52, no. 4, pp. 1007-1014, 2004.

[6] K.-C. Lee, "Frequency-domain analyses of nonlinearly loaded antenna arrays using simulated annealing algorithms," Progress in Electromagnetics Research, vol. 53, pp. 271-281, 2005.

[7] J. Robinson and Y. Rahmat-Samii, "Particle swarm optimization in electromagnetics," IEEE Transactions on Antennas and Propagation, vol. 52, no. 2, pp. 397-407, 2004.

[8] M. Behera, A. B. Sahoo, B. R. Behera, H. Pradhan, and B. B. Mangaraj, "Optimum design of mutually coupled linear array antenna and comparison with different types of array structures," in Proceedings of the 2nd International Conference on Communication and Signal Processing (ICCSP '13), pp. 3943, Melmaruvathur, India, April 2013.

[9] S.-L. Cheng and C. Hwang, "Optimal approximation of linear systems by a differential evolution algorithm," IEEE Transactions on Systems, Man, and Cybernetics Part A: Systems and Humans, vol. 31, no. 6, pp. 698-707, 2001.

[10] B. B. Mangaraj, I. S. Misra, and S. K. Sanyal, "Application of bacteria foraging algorithm in designing log-periodic dipole array for entire UHF TV spectrum," International Journal of RF and Microwave Computer-Aided Engineering, vol. 23, no. 2, pp. 157-171, 2013.

[11] B. B. Mangaraj, I. S. Misra, and A. Barisal, "Optimizing included angle of symmetrical $\mathrm{V}$ dipoles for higher directivity using bacteria foraging optimization algorithm," Progress in Electromagnetics Research B, vol. 3, pp. 295-314, 2008.

[12] B. B. Mangaraj, I. S. Misra, and S. K. Sanyal, "Application of bacteria foraging algorithm for the design optimization of multiobjective Yagi-Uda array," International Journal of RF and Microwave Computer Aided Engineering, vol. 21, no. 1, pp. 2535, 2011.

[13] M. Tripathy and S. Mishra, "Bacteria foraging-based solution to optimize both real power loss and voltage stability limit," IEEE Transactions on Power Systems, vol. 22, no. 1, pp. 240-248, 2007.

[14] K. M. Passino, Biomimicry of bacterial foraging for distributed optimization, Princeton University Press, Princeton, NJ, USA, 2001.

[15] K. M. Passino, "Biomimicry of bacterial foraging for distributed optimization and control," IEEE Control Systems Magazine, vol. 22, no. 3, pp. 52-67, 2002.

[16] U. Alon, M. G. Surette, N. Barkai, and S. Leibler, "Robustness in bacterial chemotaxis," Nature, vol. 397 , no. 6715, pp. 168-171, 1999. 
[17] C. A. Balanis, Antenna Theory: Analysis and Design, John Wiley \& Sons, New York, NY, USA, 3rd edition, 2005.

[18] E. A. Jones and W. T. Joines, "Design of Yagi-Uda antennas using genetic algorithms," IEEE Transactions on Antennas and Propagation, vol. 45, no. 9, pp. 1386-1392, 1997. 

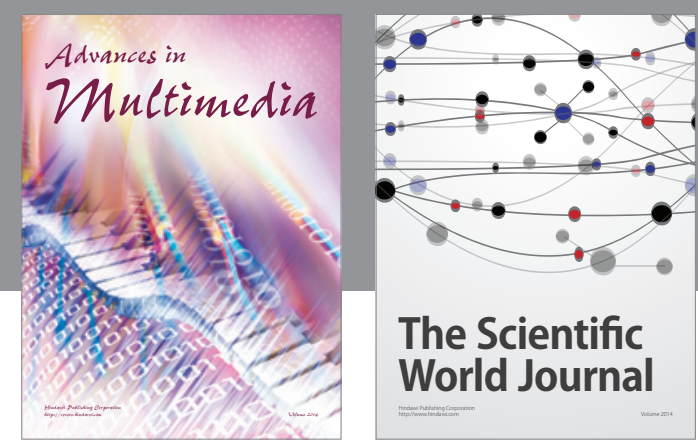

The Scientific World Journal
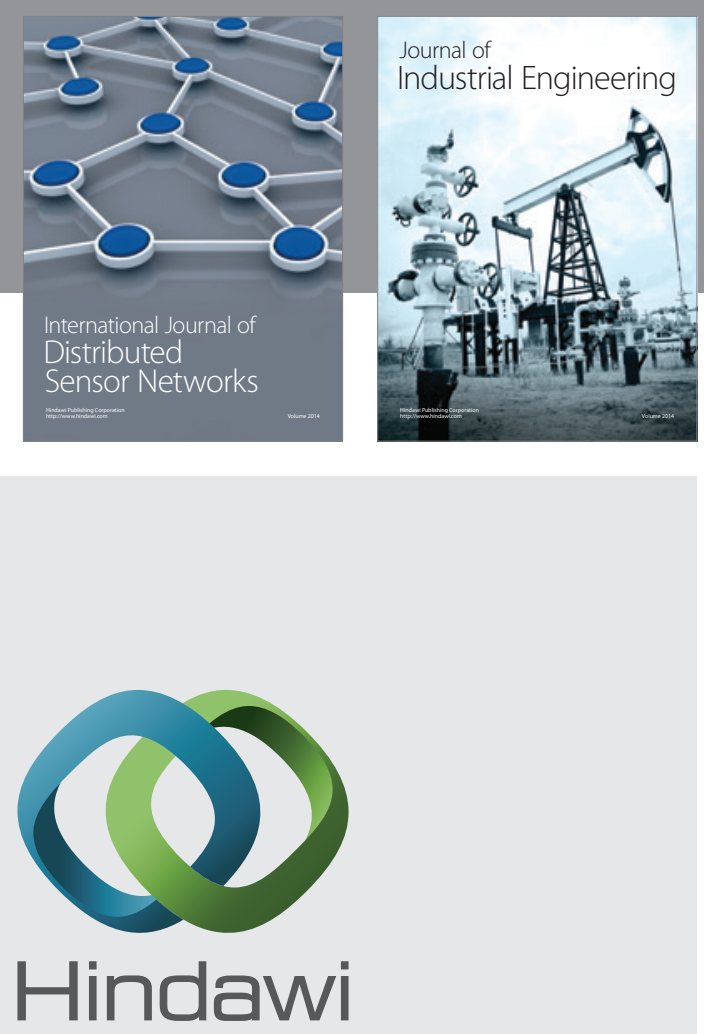

Submit your manuscripts at

http://www.hindawi.com

\section{Computer Networks} and Communications
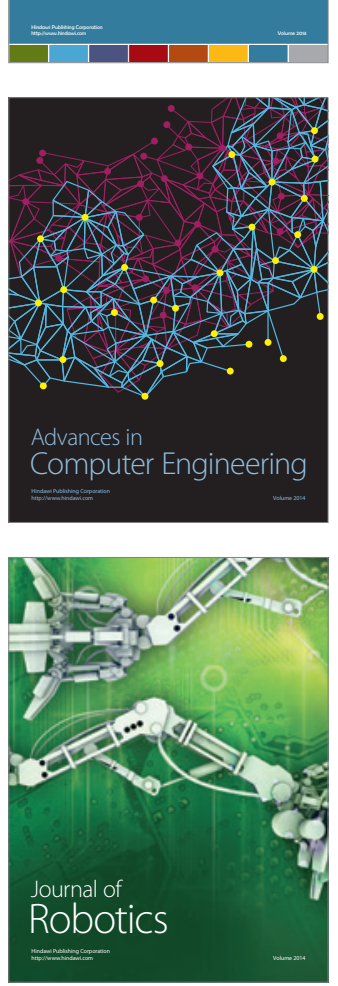
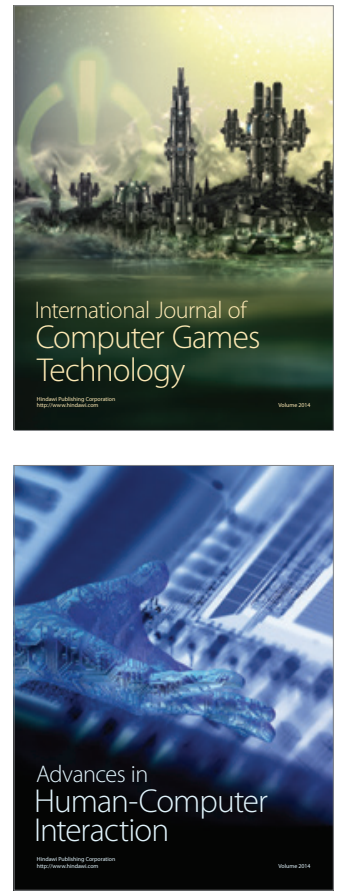
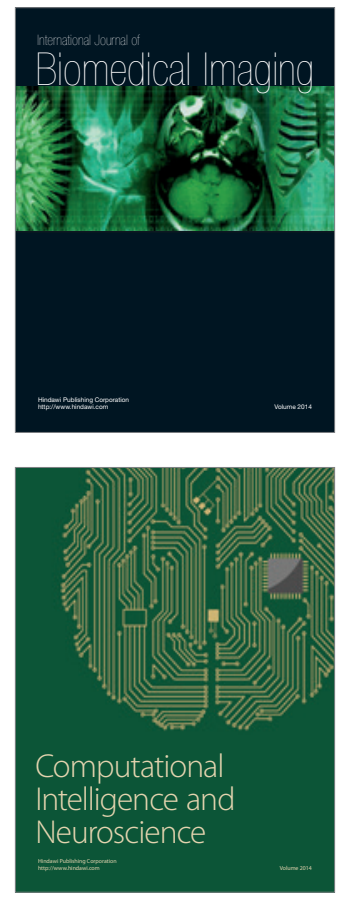
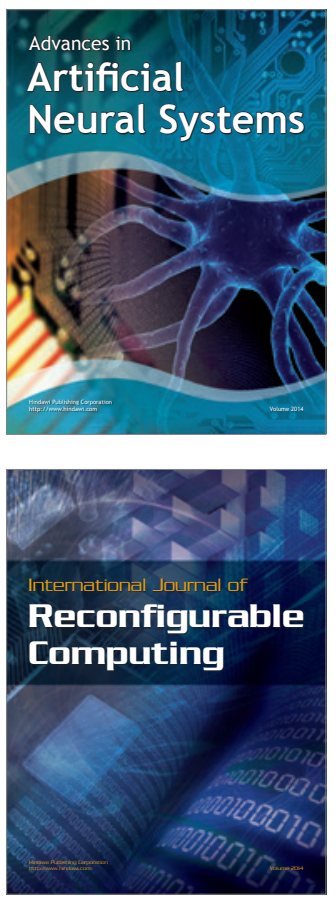
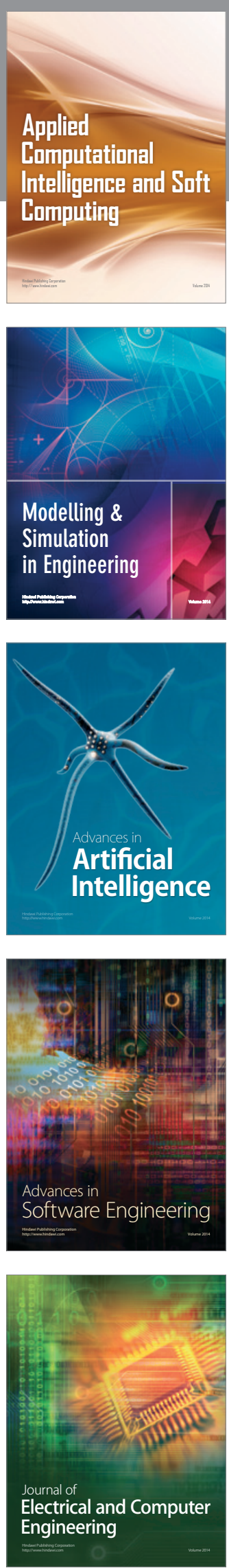